\title{
British Orthodontic Conference 2018
}

The 31st annual British Orthodontic Conference (BOC) organised by the British Orthodontic Society (BOS) took place between 27 and 29 September 2018. The conference was held in London for the first time at the Queen Elizabeth II Conference Centre.

Orthodontics $360^{\circ}$ was the theme set for the conference, with strong emphasis on all aspects to orthodontics and interdisciplinary care.

The conference was opened by Richard Jones, Chair of BOC 2018. The main scientific programme started with University Teachers Group research sessions, involving passionate orthodontic StRs (Specialty Training Registrars) showcasing their involvement in the latest orthodontic research and technological advances.

Martyn Cobourne, Professor of Orthodontics, gave the Chapman Prize 2018 presentation about the impact of obesity on orthodontic tooth movements in adolescents.

Padhraig Fleming, a specialist in orthodontics and $B D J$ Associate Editor, gave an insightful presentation on high-quality orthodontic evidence levels and mechanisms of accelerating tooth movements, while Kevin O’Brien, Professor of Orthodontics, gave a refreshing talk on social media and orthodontics.

In parallel to the main scientific programme, NHS Commissioning series were simultaneously running throughout the day, covering primary contracts and updates from NHSBSA Dental Services.

The second day was interdisciplinary focused. Sarah Good kicked off the day by discussing the orthodontic-periodontal interface, while Raman Aulakh talked about adult orthodontics and aesthetic dentistry.

The session also involved talks regarding classifications and orthodontic handling of TMD (temporomandibular disorder) patients, aesthetic Smile Design, and orthognathic surgery.

Trauma and orthodontics were discussed by Nicola Atack and Helen Rodd, Professor of Paediatric Dentistry. Jonathan Sandler, Professor of Orthodontics, concluded the day by discussing malocclusions and periodontal disease and the role of the orthodontist.

The third day saw a variety of Academic Rising Stars and Clinical Pearls presentations.

The day held the exciting new addition to the conference - the OrthoTED session
(Technology, Education and Design). OrthoTEDs offered a variety of 20-minute presentations that aimed to improve the education and practice of orthodontics. Prizes and acknowledgement were announced. A member of the BOS's Education Committee, David Waring was announced as BOC 2019 Chairman.

This year, the BOC has introduced new catch-up sessions in which video recordings of many presentations were shown at a later time during the conference for those who were unable to attend the live sessions.

I had a poster presentation at the conference and had real pleasure meeting inspirational leaders of the orthodontic world. Overall, I found this year's conference to be well structured and very informative.

Details of BOC 2019 Glasgow (19-21 September 2019) can be found at https:// www.bos.org.uk/News-and-Events/ Events-Meetings.

By Fahad Alawsi, DCT2 Paediatric Dentistry/ Orthodontics University Dental Hospital of Manchester, OMFS/SCD/Restorative Dentistry Royal Preston Hospital

\section{Dental cycle charity effort raises almost $£ 40,000$}

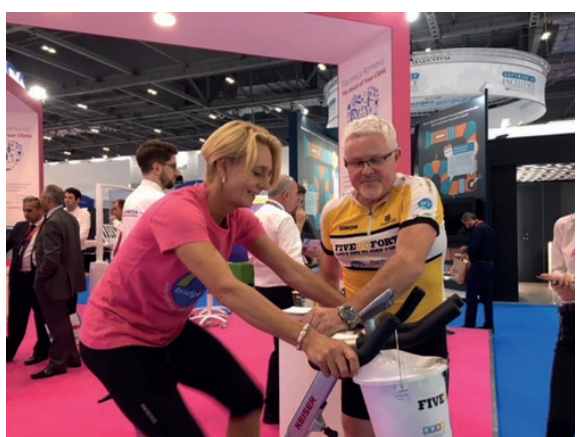

Sara Hurley, Chief Dental Officer (CDO) for England

Five dental business consultants cycled more than 1,000 miles in 15 days from Land's End to John O'Groats in September to raise funds for three charities - Cancer Research UK, Bridge2Aid and BrushUpUK.
Chris Barrow, Les Jones, Sheila Scott, Simon Tucker and Ashley Latter raised $£ 38,064$ (including Gift Aid). The team was sponsored by Practice Plan, Dental Sky, Wesleyan and Dental Focus.

The campaign received an additional boost when visitors to the recent BDIA Dental Showcase event held in London in October 2018 were encouraged to do their bit on a spin bike.

Sara Hurley, CDO for England, also joined in at the event to encourage last minute donations.

Simon Tucker, Chief Executive Officer for Dental Sky, said: 'We're all getting older and sadly many of us will experience cancer in our lives. The NHS has launched a huge campaign highlighting the danger of obesity and cancer, so it made sense to do something physical which would raise awareness of the link between the two as well as raising funds for cancer research.

'Dental professionals are almost certainly aware of the amazing work Bridge2Aid does in Africa training local medical officers to carry out basic dentistry. As we all work in the dental sector, it was an obvious choice to make them a beneficiary of our efforts as too was BrushUpUK, a charity that provides dental education and guidance to vulnerable groups in society'.

A fundraising page will remain open for anyone who would like to support the challenge - www.fivegoforth.co.uk. 